A. Hauschild

S. Eiling

\section{Bestimmung der Tumormarker S-100B und MIA im Blut von Melanompatienten}

\author{
Determination of Tumor Markers S-100B and MIA in the Blood \\ of Melanoma Patients
}

\section{Zusammenfassung}

Bei den meisten soliden Tumoren können mehr oder weniger spezifische tumorassoziierte Moleküle im Blut nachgewiesen werden. Beim malignen Melanom stehen mit den kommerziell erhältlichen Nachweisverfahren zur Quantifizierung von Protein S-100B (Sangtec100-LIA) und „melanoma-inhibitory activity“ (MIA-ELISA) gleich zwei Testkits zur Verfügung. Vergleichende Untersuchungen haben gezeigt, dass bei einer Spezifität von $95 \%$ etwa 70 bis $90 \%$ aller Patienten mit fernmetastasiertem Melanom durch erhöhte Serumwerte von S-100B bzw. MIA auffallen. Beide Tumormarker-Werte im Blut korrelieren stadienabhängig sehr gut mit der Prognose von Melanompatienten, so dass ein effektives Therapiemonitoring möglich wird. Durch S-100B- und MIA-Bestimmungen können jedoch ganz offensichtlich keine okkulten Mikrometastasen entdeckt werden, so dass ihre Verwendung nur bei Patienten mit erhöhtem Metastasierungsrisiko sinnvoll erscheint.

\section{Abstract}

In the majority of solid neoplasia a detection of tumor-associated molecules in blood is possible. In malignant melanoma, two commercially available test systems to quantify melanoma-associated proteins S-100B (Sangtec 100-LIA), and melanoma inhibitory activity (MIA-ELISA) can be used in routine. Comparative studies revealed that at a specificity of $95 \%$ about 70 to $90 \%$ of advanced metastatic melanoma patients will demonstrate elevated S-100B or MIA serum concentrations. Both markers show a stage-dependent correlation of serum values with prognosis of the patients. Thus, therapy monitoring using these melanoma markers seems to be attractive. Unfortunately, detection of occult micrometastasis is obviously unrealistic. The use of S-100 and MIA detection in serum should be limited to patients with a substantial risk of metastasis.

\section{Einleitung}

Unter Tumormarkern versteht man tumorassoziierte Erkennungsmoleküle (z.B. Proteine oder Peptide), die sich durch unterschiedliche Methoden nachweisen lassen und einen möglichst hohen Wert für das Screening, die Differenzialdiagnostik, das Routine-Staging oder auch das Therapie-Monitoring von Tumor-Patienten besitzen [14]. Der klinische Wert derartiger Tumormarker hängt entscheidend von deren Spezifität (möglichst wenig falsch positive Resultate), aber auch der Sensitivität (möglichst wenig falsch negative Resultate) ab. Eine weitere zu beachtende Größe bei der Beurteilung von Tumormarkern ist der so genannte „prädiktive Wert“ eines Markers, der eine Art Validi- tätskontrolle darstellt $[12,20]$. Leider erfüllt auch heute unter allen bekannten Markern für die verschiedenen Tumorentitäten kein Marker die Idealvorstellung einer 100\%igen Spezifität sowie einer gleichfalls hohen Sensitivität, die idealerweise auch $100 \%$ betragen sollte. Vielleicht erreicht das prostataspezifische Antigen (PSA) - ein relativ organspezifischer Marker, der einen breiten Einsatz in der Routine beim Prostatakarzinom eingenommen hat - am ehesten die Kriterien eines unabhängigen wertvollen Tumormarkers. Leider ist auch der PSA-Spiegel im Blut bei nichtmalignen Erkrankungen, wie z.B. der Prostatahyperplasie, erhöht, so dass eine Erhöhung des PSA-Spiegels nicht automatisch ein Hinweis für eine Malignität bedeutet [5]. 
In einem Übersichtsreferat [18] zum allgemeinen Wert von Tumormarkern in der klinischen Onkologie ohne besondere Betonung des malignen Melanoms kommt Hossfeld 1996 zu dem Schluss, dass es sehr viele nutzlose Tumormarker gibt und leider würde nicht immer hinterfragt werden, ob die Bestimmung des Tumormarkers wirklich eine valide Aussage bezüglich der Prognose oder des weiteren Therapie-Procederes für die Patienten bedeutet. Als Beispiel führt Hossfeld an, dass bekannt ist, dass eine gesunde Person, bei der 13 Tumormarker im Blut bestimmt werden, eine Chance von nur $51 \%$ hat, auch als wirklich "gesund“ klassifiziert zu werden. Somit würden fast $50 \%$ falsch positive Resultate durch eine derartige Multi-Marker-Bestimmung zustande kommen [24]. Ein weiterer Faktor sind die teilweise erheblichen Kosten, die durch eine Tumormarker-Bestimmung im Blut entstehen [18].

Im Folgenden soll zur Quantifizierung der melanomassoziierten Moleküle Protein S-100B und Melanoma-inhibitory activity (MIA), die durch Tumorzellen synthetisiert und/oder sezerniert werden und somit prinzipiell als Tumormarker des Melanoms dienen könnten, Stellung genommen werden. Dabei wird besonders der Sensitivität und der Spezifität der beiden Melanommarker-Moleküle Rechnung getragen.

\section{Protein S 100B}

Den Begriff „S-100“ prägte Moore [23] in der Erstbeschreibung eines kalziumbindenden Proteins, das aus Rinderhirn stammte und bei neutralem $\mathrm{pH}$ in $100 \%$ Ammoniumsulfat löslich war. Heute weiß man, dass die S-100-Proteinfamilie mehrere Subgruppen besitzt [25], von denen nur die Untereinheit S-100B durch einen kommerziell erhältlichen luminoimmunometrischen Assay (Sangtec 100-B LIA ${ }^{\circledR}$ ) in der Routine bestimmt werden kann.

Die Funktionen der S100-Proteinfamilie sind immer noch nicht genau bekannt, aber in den letzten Jahren mehrt sich das Wissen um die Biologie dieser kalziumabhängigen Proteine beträchtlich. Insgesamt sind 19 Mitglieder der S-100-Familie, die alle eine distinkte Gewebeexpression aufweisen, beschrieben worden. Die Hauptfunktionen [9] werden in der Beeinflussung des intrazellu- lären Kalziumstoffwechsels im Sinne eines Kalziumrezeptorproteins gesehen, obwohl auch extrazelluläre Funktionseigenschaften bekannt sind. Der wahrscheinlich in der Onkologie bedeutsamste Effekt von Protein S-100 liegt in der in vitro nachgewiesenen Hemmung der kalziumabhängigen Phosphorylierung von dem Tumorsuppressorgen p 53 durch Proteinkinase C. S-100B wird nicht nur von Melanomzellen und benignen Melanozyten, sondern auch von Nervengewebe und epidermalen LangerhansZellen [29] wie auch von interdigitierenden Retikulumzellen der Haut und Schleimhaut exprimiert. Darüber hinaus zeigt sich auch eine Expression in Chondrozyten, Merkelzellen sowie den assoziierten neoplastischen Varianten dieser Zellgruppen (Tab.1).

Ghanem u. Mitarb. [11] simulierten an einem In-vitro-Modell den Zelltod von Melanomzellen und untersuchten in vivo Melanompatienten, die sich einer isolierten Extremitätenperfusion mit Tumornekrosefaktor alpha und Melphalan unterzogen hatten. Die belgische Arbeitsgruppe war daran interessiert, wie das intrazytoplasmatische Protein S-100 in hohen Konzentrationen in das Blut gelangen kann. Die experimentellen Untersuchungen zeigten, dass durch den Untergang von Melanom- und Endothelzellen eine Freisetzung von S-100B in die Blutzirkulation erfolgen kann. Die geschätzte Halbwertszeit von endogenem S-100B würde ca. 30 min betragen [11].

Die S-100B-Blutkonzentrationen sind erheblich von der Blutaufbearbeitung und -lagerung nach Entnahme abhängig. Kürzlich konnte eine Mannheimer Arbeitsgruppe zeigen, dass Vollblut und Serum, das drei Stunden bei Zimmertemperatur gelagert wurde, deutlich höhere S-100B-Werte als sofort bearbeitetes Blut aufwies [8].

Klarere Arbeitsanweisungen zur Blutverarbeitung und -versendung sollten in naher Zukunft von Herstellern und Anwendern gemeinsam erarbeitet werden.

\section{Prognostische Bedeutung}

Eine Deutsche [13] und eine Schweizer Arbeitsgruppe [17] sammelten Serumproben von 126 bzw. 73 Melanompatienten und

Tab. 1 Übersicht zur Charakterisierung und klinischem Einsatz der Tumormarker-Moleküle S-100B und MIA

\begin{tabular}{|c|c|c|}
\hline & $S-100 B$ & MIA \\
\hline Molekulargewicht & $21 \mathrm{kD}$ & $11 \mathrm{kD}$ \\
\hline Expressionsverhalten & $\begin{array}{l}\text { Melanomzellen, Melanozyten } \\
\text { Chondrozyten, Merkelzellen } \\
\text { Nervengewebe } \\
\text { dendritische Zellen }\end{array}$ & $\begin{array}{l}\text { Melanomzellen } \\
\text { Chondrozyten } \\
\text { Melanozytäre Nävi } \\
\text { (andere: ??) }\end{array}$ \\
\hline Nachweisverfahren (Blut) & Luminoimmunometrischer Assay (Sangtec 100-LIA) & MIA-ELISA (nicht-radioaktiv) \\
\hline empfohlen vom Hersteller & $0,12 \mu \mathrm{g} / \mathrm{l}$ & $6,5 \mu \mathrm{g} / \mathrm{l}$ \\
\hline eigene Empfehlungen & $0,20 \mu \mathrm{g} / \mathrm{l}$ & $10,0 \mu \mathrm{g} / \mathrm{l}$ \\
\hline \multicolumn{3}{|l|}{ Sensitivitäten } \\
\hline Stad. I/II & $0-10 \%$ & $0-20 \%$ \\
\hline Stad. III & $30-50 \%$ & $30-50 \%$ \\
\hline
\end{tabular}


konnten erhöhte S-100-Spiegel in einer stadiumabhängigen Verteilung feststellen (Stadium I/II: 1,3\%/4,0\%; Stadium III: 8,7\%/21,4\%; Stadium IV: 73,9\%/79,4\%). Bonfrer et al. bestätigten die hohe Sensitivität von S-100B bei fernmetastasierten Melanompatienten mit dem luminoimmunometrischen Nachweisverfahren (LIA-mat) [1].

Diese Resultate, die eine hohe Sensitivität des S-100B-Tumormarkers im Stadium der Fernmetastasierung nahelegen, werden unterstützt durch umfangreiche Untersuchungen einer Stockholmer Arbeitsgruppe [27] an 643 Melanompatienten. Hier zeigte sich ein statistisch hoch signifikanter Unterschied zwischen S-100B-positiven im Vergleich zu S-100B-negativen Patienten im Serum hinsichtlich der Überlebenszeit. Die schwedische Arbeitsgruppe sieht den S-100B-Test im Serum als einen unabhängigen prognostischen Faktor für Melanompatienten und legt somit die Bestimmung in der Routine nahe [27].

In einer weiteren Studie an 270 Patienten kommen Miliotes $\mathrm{u}$. Mitarb. zu dem Schluss, dass die S100B-Bestimmung im Serum sowohl ein signifikanter Prädiktor für ein Rezidiv als auch für die Gesamtüberlebenszeit ist. Die Autoren empfehlen eine prospektive Untersuchung während angewandter Therapiestudien bei Patienten, die nach den vorliegenden Staging-Untersuchungen „tumorfrei“ sind und eines engmaschigen Follow-ups bedürfen [22].

In unserer eigenen Arbeitsgruppe [15] konnte zunächst anhand von 1339 Serumproben von 412 verschiedenen Melanompatienten und 107 Kontrollpatienten festgestellt werden, dass 1,7\% der Patienten im Stadium mit einem Primärtumor ohne Hinweis für eine Metastasierung, 19,2\% der Patienten mit lokoregionärer Metastasierung sowie $67,9 \%$ der Patienten mit Fernmetastasierung erhöhte S-100B-Werte im Serum aufwiesen. Hierbei zeigten sich statistisch signifikante Unterschiede zwischen Primärtumor- und dem Stadium der Metastasierung. Die daraus errechnete Überlebenszeit während der 30-monatigen Nachbeobachtung der Tumorpatienten ergab, dass Patienten mit Normalwerten für S-100B im Serum eine mediane Überlebenszeit von 28 Monaten aufwiesen, während Patienten mit erhöhten S-100BWerten im Serum lediglich 6 Monate überlebten. Sowohl im Stadium der regionären Metastasierung (Stadium III) als auch im Stadium der Fernmetastasierung erwies sich der S-100B-Wert im Serum als ein statistisch signifikanter Prognoseparameter [19].

\section{Therapiemonitoring}

Eine Untersuchung zum Monitoring von Patienten mit metastasiertem malignen Melanom während 77 Therapiezyklen bei 64 unterschiedlichen Patienten konnte zeigen, dass das Therapieergebnis (Progress oder Remission der Metastasen) anhand des Verlaufes der S-100B-Werte im Serum vorhersagbar ist [16]. Bei Patienten, die auf eine Therapie mit einer stabilisierten Erkrankung oder einer Tumorregression reagierten, fiel der S-100BWert im Serum parallel dazu in $98 \%$ aller Fälle (39 von 40 Patienten). Bei Patienten, die eine Progredienz der Tumorerkrankung aufwiesen, zeigte sich in $84 \%$ aller Fälle (31 von 37 Patienten) ein steigender S-100B-Wert. Bereits bei einer Zwischenanalyse nach 4 Wochen war bei der Mehrzahl der Patienten das Therapieergebnis vorherzusagen [16].
In der Untersuchung einer Mannheimer Arbeitsgruppe wurde anhand von 271 Serumproben von 65 Melanompatienten aufgezeigt, dass der klinische Verlauf im Sinne einer Progression oder Regression der Metastasierung in $81,5 \%$ der Fälle mit den S-100-Werten direkt korrelierte [7].

\section{Melanoma Inhibitory Activity (MIA)}

Das Protein Melanoma Inhibitory Activity wurde vor einigen Jahren aus einer langsam wachsenden Metastase eines Patienten mit malignem Melanom isoliert. Die Entdeckung von MIA und dessen spätere Sequenzierung und Klonierung haben das Verständnis für die Bedeutung der Adhäsion im Rahmen der Tumorgenese maligner Melanome vertieft. Der Verlust der Haftung zwischen den Zellen ist ein entscheidender Schritt im Frühstadium der Metastasierung. Für die Anheftung von Tumorzellen an die extrazelluläre Matrix sorgen Zelloberflächenmoleküle aus der Integrin-Rezeptorfamilie. MIA inhibiert spezifisch die Anbindung von Melanomzellen in Fibronektin und Laminin. Es kommt zu einer direkten Bindung von MIA und Matrixproteinen. Diese Mechanismen erklären, warum MIA die Fähigkeit besitzt, Melanomzellen in vitro im Wachstum zu hemmen und führten zur Namensgebung „Melanoma Inhibitory Activity“ (Übersicht bei [3]).

Eine Expression von MIA-mRNA und Protein konnte bisher in fast allen Melanomen nachgewiesen werden. Melanozyten aus normaler Haut exprimieren im Gegensatz zu Melanomzellen kein MIA, melanozytäre Nävi zeigten eine schwache bis mittelstarke Expression (Tab.1). Zunächst wurde davon ausgegangen, dass es neben Melanomen und reifen Chondrozyten keine Gewebe gibt, die MIA exprimieren [3,4].

De Vries u. Mitarb. konnten 1999 jedoch zeigen, dass MIA-mRNA neben der Expression in 16 von 19 untersuchten Melanomzelllinien auch von sieben Nicht-Melanomzelllinien exprimiert wird. Darüber hinaus fand sich in allen untersuchten Blutproben von Melanompatienten, aber auch in Blutproben von Nicht-Melanompatienten und gesunden Blutspendern eine MIA-Expression [30]. Die Autoren gehen davon aus, dass geringe Mengen von MIA-Transkripten auch in Normalgewebe und weiteren NichtMelanom-Tumoren aufzufinden sind, und der Marker daher nicht geeignet ist, um für die molekularbiologische Detektion von Melanomzellen im peripheren Blut eingesetzt zu werden.

Die Blutaufbearbeitung und -lagerung scheint für die Bestimmung von MIA mittels eines nicht-radioaktiven ELISA relativ unproblematisch zu sein. Temperaturänderungen der Umgebung scheinen MIA-Werte nicht zu verändern. Ein zirkadianer Rhythmus der MIA-Konzentration im Blut ist nicht festzustellen [8].

In einer ersten monozentrischen Untersuchung [2] zeigte sich bei allen Patienten (100\%ige Sensitivität) mit Melanommetastasen (Stadium III/IV) ein erhöhter MIA-Wert im Serum. Als Referenzwert wurde die 95\%-Perzentile von gesunden Kontrollpatienten angesehen. Die weitere Auswertung ergab, dass der MIASpiegel im Serum von gesunden Probanden altersunabhängig war und als oberer Normwert 6,5 ng/ml ermittelt wurden. 30\% der Melanompatienten im Stadium I und 23\% im Stadium II wie- 
sen erhöhte Serumspiegel ohne eine direkte Korrelation mit der Tumordicke auf [2].

Nachfolgend wurden in einer Untersuchung mehrerer Zentren [4] von 259 Patienten mit metastasiertem malignen Melanom 419 Serumproben sowohl prä- als auch postoperativ entnommen und untersucht. Die Bestimmung von MIA im Serum erfolgte mittels einer modifizierten ELISA-Technik. Positive MIA-Werte im Serum wurden präoperativ bei 22 der 37 Stadium-III-Patienten (59\%) sowie 157 der 176 (89\%) der Stadium-IV-Patienten gefunden. Die Untersuchung postoperativ entnommener Seren nach vollständiger Tumorentfernung ergab in 31,8\% der Fälle (Stadium III) bzw. 21 \% der Fälle (Stadium IV) erhöhte Serumwerte [4].

Eine Münchener Untersuchung an 830 Blutproben von 326 Melanompatienten zeigte, dass bei 5,6\% der Patienten mit Primärtumoren im Gegensatz zu 60\% mit lokoregionären Metastasen (Stad. III) bzw. 89,5\% mit Fernmetastasen (Stad. IV) MIA-Erhöhungen oberhalb des hier verwendeten Cut-off-Wertes von $9,8 \mathrm{ng} / \mathrm{ml} \mathrm{zu}$ finden waren. Bei dieser Untersuchung fiel auf, dass die Patienten mit niedrigen MIA-Werten zumeist vorher operiert, bestrahlt oder chemotherapeutisch behandelt waren und insgesamt eine bessere Prognose aufwiesen [28].

Vergleichende Untersuchungen zu S-100B und MIA im Blut von Melanompatienten

Bereits in der ersten Publikation [2] zu dem neuen Melanom-Tumormarker MIA konnten Bosserhoff et al. neben den MIA-Untersuchungen an einer großen Zahl von Melanompatienten auch noch an einem kleineren Patientenkollektiv S-100B-Serumkonzentrationen bestimmen. Während alle Patienten im Stadium III/ IV MIA-positiv waren, zeigten lediglich 30 von 49 Seren (61,2\%) für S-100B erhöhte Werte. Die Autoren stellten kritisch die Spezifität des Tumormarkers S-100B für das maligne Melanom infrage, da aus dem Kontrollpatientenkollektiv 20\% der Patienten mit Sepsis, 16\% der Patienten mit Gliomen sowie 5\% der Patienten mit fortgeschrittenen Karzinomen erhöhte S-100B-Serumkonzentrationen aufwiesen. Zusammenfassend stellte diese und eine weitere Untersuchung der gleichen Arbeitsgruppe fest, dass MIA dem Marker S-100B sowohl hinsichtlich der Sensitivität als auch Spezifität überlegen zu sein scheint $[2,19]$.

Weitere Untersuchungen zu diesem Themenkomplex folgten. Eine Monitoring-Untersuchung in verschiedenen Tumorstadien bei insgesamt 791 Serumproben von 87 Melanom-Patienten und 158 Kontrollpatienten zeigte eine leichte Überlegenheit von S-100B im Vergleich zu MIA [26].

Ähnliche Ergebnisse wurden von Djukanovic et al. erhoben. S-100B und MIA zeigten annähernd gleiche Ergebnisse in Bezug auf die klinische Korrelation (Progression oder Regression der Metastasen) mit Veränderungen von MIA- bzw. S-100-Serumkonzentrationen [7]. In einer weiteren umfangreichen Untersuchung aus Ulm konnte der hohe Stellenwert von S-100B im Vergleich zu MIA und der LDH aufgezeigt werden. Krähn et al. fassten zusammen, dass S-100B derzeit der attraktivste Tumormarker für das metastasierte maligne Melanom sei [21].
Eine Heidelberger Studie zu S-100B, MIA und der Laktatdehydrogenase (LDH) zur Diskriminierung von Patienten mit progredienter Grunderkrankung von Patienten mit nicht-progredienter Grunderkrankung, zeigte eine nahezu identische Sensitivität und Spezifität für S-100B und MIA. Allerdings war in dieser Untersuchung die LDH der insgesamt am besten mit dem Krankheitsverlauf korrelierende Laborparameter [6].

\section{Ausblick}

Die Untersuchung von tumorassoziierten Molekülen im Blut („Tumormarkern“) hat sich bei einer Reihe von soliden malignen Tumoren insbesondere in der Nachsorge und im Therapiemonitoring bewährt. In der Schweiz hat die Vielzahl von überzeugenden Untersuchungen zu den Melanom-Tumormarkern S-100B und MIA dazu geführt, beide Marker als einzige optionale Blutentnahmen in die Empfehlungen zur Nachsorge bei malignen Melanomen bei Patienten mit mehr als $1 \mathrm{~mm}$ dicken Primärtumoren und/oder Lymphknotenmetastasen aufzunehmen [10]. Auf die Untersuchung weiterer laborchemischer Parameter wird hingegen verzichtet. Mit den kommerziell erhältlichen Testkits für S-100B und MIA stehen standardisierte Bestimmungsverfahren zur Verfügung, die in Routinelabors durchgeführt werden können. Sowohl S-100B als auch MIA scheinen bei einer Spezifität von $95 \%$ eine Sensitivität zwischen 70 und $90 \%$ für das fernmetastasierte Melanom aufzuweisen. Dies bedeutet, dass die Mehrzahl der Patienten mit metastasierten Melanomen mittels einer einfachen serologischen Untersuchung identifiziert werden können. Die Rate falsch positiver Befunde ist dabei definitionsgemäß mit etwa 5\% relativ gering. Sowohl S-100B als auch MIA sind aber ganz offensichtlich nicht geeignet, eine sehr frühe Metastasierung („Mikrometastasierung“) zu erkennen.

In viele nationale und internationale Studienprojekte wurden die Markermoleküle S-100B und/oder MIA als „Surrogate Marker“ inzwischen aufgenommen. Eine großangelegte deutsche Studie zum Wert der Nachsorgeuntersuchungen beim malignen Melanom hat S-100B als Laborparameter eingeschlossen. Diese Untersuchung soll klären, welchen Wert die körperliche Untersuchung, Routine-Blutabnahmen und die apparative Diagnostik in der Melanomnachsorge aufweisen. Hierzu sollen in den Jahren 2002 und 2003 an den Universitäts-Hautkliniken in Tübingen, Mannheim und Kiel etwa 4000 Melanompatienten mit unterschiedlichen Nachsorgeschemata untersucht werden. Mit Ergebnissen der prospektiven Untersuchung dürfte aber erst im Jahr $2005 \mathrm{zu}$ rechnen sein.

\section{Literatur}

${ }^{1}$ Bonfrer JM, Korse CM, Nieweg OE, Rankin EM. The luminescence immunoassay S-100: a sensitive test to measure circulating S-100B: its prognostic value in malignant melanoma. Br J Cancer 1998; 77: $2210-2214$

2 Bosserhoff AK, Kaufmann M, Kaluza B, Bartke I, Zirngibl H, Hein R Stolz W, Buettner R. Melanoma-inhibiting activity, a novel serum marker for progression of malignant melanoma. Cancer Res 1997; 57: $3149-3153$

${ }^{3}$ Bosserhoff AK, Golob M, Buettner R, Landthaler M, Hein R. MIA (Melanoma inhibitory activity). Hautarzt 1998; 49: $762-769$

${ }^{4}$ Bosserhoff AK, Hauschild A, Hein R, Schadendorf D, Stockfleth E, Bogenrieder T, Landthaler M, Buettner R, Stolz W. Elevated MIA serum 
levels are of relevance for management of metastasized malignant melanomas: results of a German multicenter study. J Invest Dermatol 2000; 114: 395-396

${ }^{5}$ Chu TM. Prostate-specific antigen. In: Sell S (Ed). Serological Cancer Markers. Totowa: Humana Press, 1992: 99-115

${ }^{6}$ Deichmann M, Benner A, Bock M, Jäckel A, Uhl K, Waldmann V, Näher H. S100- $\beta$, melanoma-inhibiting activity, and lactate dehydrogenase discriminate progressive from nonprogressive American Joint Committee on Cancer stage IV melanoma. J Clin Oncol 1999; 17: $1891-1896$

7 Djukanovic D, Hofmann U, Sucker A, Rittgen W, Schadendorf D. Comparison of S-100 protein and MIA protein as serum marker for malignant melanoma. Anticancer Res 2000; 20: 2203 - 2207

8 Djukanovic D, Hofmann U, Sucker A, Schadendorf D. Melanoma tumor markers S100B and MIA: evaluation of stability in serum and blood upon storage an processing. Br J Dermatol 2001; 145: 1 -3

${ }^{9}$ Donato R. Functional roles of S100 proteins, calcium-binding proteins of EF-hand type. Biochim Biophys Acta 1999; 1450: 191 -231

${ }^{10}$ Dummer R, Bosch U, Panizzon R, Bloch PH, Burg G. Task force 'Skin Cancer'. Swiss National Program against Cancer. Swiss Cancer League. Swiss guidelines for the treatment and follow-up of cutaneous melanoma. Dermatology 2001; 203: 75-80

${ }^{11}$ Ghanem G, Loir B, Morandini R, Sales F, Lienard D, Eggermont A, Lejeune $\mathrm{F}$. On the release and half-life of $\mathrm{S} 100 \mathrm{~B}$ protein in the peripheral blood of melanoma patients. Int J Cancer 2001; 94: 586-590

12 Gläser R. Tumormarker des malignen Melanoms. In: Garbe C, Dummer R, Kaufmann R, Tilgen W (Hrsg). Dermatologische Onkologie. Berlin: Springer, 1997: $324-329$

${ }^{13}$ Guo HB, Stoffel-Wagner B, Bierwirth T, Mezger J, Klingmüller D. Clinical significance of serum S100 in metastatic malignant melanoma. Eur J Cancer 1995; 31: $924-928$

${ }^{14}$ Hauschild A. The use of serological tumor markers for malignant melanoma. Onkologie 1997; 20: 462 - 465

${ }^{15}$ Hauschild A, Engel G, Brenner W, Gläser R, Monig H, Henze E, Christophers E. S100B protein detection in serum is a significant prognostic factor in metastatic melanoma. Oncology 1999; 566: 338 - 344

${ }^{16}$ Hauschild A, Engel G, Brenner W, Gläser R, Mönig H, Henze E, Christophers E. Predictive value of serum S100B for monitoring patients with metastatic melanoma during chemotherapy and/or immunotherapy. Br J Dermatol 1999; 144: 1065 - 1071

${ }^{17}$ Henze G, Dummer R, Joller-Jemelka HI, Böni R, Burg G. Serum S100 - a marker for disease monitoring in metastatic melanoma. Dermatology 1997; 194: $208-212$
${ }^{18}$ Hossfeld DK. Tumor marker terrorism. Educational Book; 21. Congress European Society of Medical Oncology (ESMO); 1.-5.11.1996. Wien: 1996: $191-192$

19 Juergensen A, Holzapfel U, Hein R, Stolz W, Buettner R, Bosserhoff A. Comparison of two prognostic markers for malignant melanoma: MIA and S100 beta. Tumour Biol 2001; 22: 54-58

${ }^{20}$ Klapdor R. Überlegungen zu einem sinnvollen Einsatz von Tumormarkern. Onkologe 1995; 1: 627-633

${ }^{21}$ Krähn G, Kaskel P, Sander S, Waizenhofer PF, Wortmann S, Leiter U, Peter RU. S100 beta is a more reliable tumor marker in peripheral blood for patients with newly occured melanoma metastases compared with MIA, albumin and lactate dehydrogenase. Anticancer Res 2001; 21: $1311-1316$

22 Miliotes G, Lyman GH, Cruse CW, Puleo C, Albertini J, Rapaport D, Glass F, Fenske N, Soriano T, Cuny C, VanVoorhis N, Reintgen D. Evaluation of new putative tumor markers for melanoma. Ann Surg Oncol 1996; 3: $558-563$

${ }^{23}$ Moore BW. A soluble protein characteristic of the nervous system. Biochem Biophys Res Commun 1965; 19: 739-744

24 Pohl AL. Multiple testing with cancer markers. In: Sell S (Hrsg). Serological Cancer Markers. Totowa: Humana Press, 1992: 473-494

${ }^{25}$ Schäfer BW, Wicki R, Engelkamp D, Mattei MG, Heizman CW. Isolation of a YAC clone covering a cluster of nine $\mathrm{S} 100$ genes on human chromosome 1 q 21: Rationale for a new nomenclature of S 100 calciumbinding protein family. Genomics 1995; 25: 638-643

${ }^{26}$ Schmitz C, Brenner W, Henze E, Christophers E, Hauschild A. Comparative study on the clinical use of protein S-100B and MIA (melanoma inhibitory activity) in melanoma patients. Anticancer Res 2000; 20: 5059-5063

${ }^{27}$ von Schoultz E, Hansson LO, Djureen E, Hansson J, Kärnell R, Nilsson B, Stigbrand T, Ringborg U. Prognostic value of serum analyses of S-100 $\beta$ protein in malignant melanoma. Melanoma Res 1996; 6: 133-137

28 Stahlecker J, Gauger A, Bosserhoff A, Büttner R, Ring J, Hein R. MIA as a reliable tumor marker in the serum of patients with malignant melanoma. Anticancer Res 2000; 20: 5041 - 5044

${ }^{29}$ Ugurel S, Pföhler C, Tilgen W, Reinhold U. S100B serum protein - a new marker in the diagnosis and monitoring of Langerhans cell histiocytosis. Br J Dermatol 2000; 143: 198-233

${ }^{30}$ De Vries TJ, Fourkour A, Punt CJ, Diepstra H, Ruiter DJ, van Muijen GN. Melanoma-inhibiting activity (MIA) mRNA is not exclusively transcribed in melanoma cells: low levels of MIA mRNA are present in various cell types and in peripheral blood. Br J Cancer 1999; 81: 1066 - 1070 